\title{
Improving the seismic small-scale modelling by comparison with numerical methods
}

\author{
Damien Pageot, ${ }^{1,2}$ Donatienne Leparoux, ${ }^{1}$ Mathieu Le Feuvre, ${ }^{1}$ Olivier Durand, ${ }^{1}$ \\ Philippe Côte ${ }^{1}$ and Yann Capdeville ${ }^{3}$ \\ ${ }^{1}$ Géophysique et Évaluation Non Destructive, IFSTTAR, GERS, GeoEND, F-44344 Bouguenais, France.E-mail: damien.pageot@ifsttar.fr \\ ${ }^{2}$ Laboratoire de Planétologie et Géodynamique de Nantes, Université de Nantes, F-44000 Nantes, France \\ ${ }^{3}$ Laboratoire de Planétologie et Géodynamique de Nantes, CNRS, Université de Nantes, F-44000 Nantes, France
}

\section{SUMMAR Y}

The potential of experimental seismic modelling at reduced scale provides an intermediate step between numerical tests and geophysical campaigns on field sites. Recent technologies such as laser interferometers offer the opportunity to get data without any coupling effects. This kind of device is used in the Mesures Ultrasonores Sans Contact (MUSC) measurement bench for which an automated support system makes possible to generate multisource and multireceivers seismic data at laboratory scale. Experimental seismic modelling would become a great tool providing a value-added stage in the imaging process validation if (1) the experimental measurement chain is perfectly mastered, and thus if the experimental data are perfectly reproducible with a numerical tool, as well as if (2) the effective source is reproducible along the measurement setup. These aspects for a quantitative validation concerning devices with piezoelectrical sources and a laser interferometer have not been yet quantitatively studied in published studies. Thus, as a new stage for the experimental modelling approach, these two key issues are tackled in the proposed paper in order to precisely define the quality of the experimental small-scale data provided by the bench MUSC, which are available in the scientific community. These two steps of quantitative validation are dealt apart any imaging techniques in order to offer the opportunity to geophysicists who want to use such data (delivered as free data) of precisely knowing their quality before testing any imaging technique. First, in order to overcome the 2-D-3-D correction usually done in seismic processing when comparing 2-D numerical data with 3-D experimental measurement, we quantitatively refined the comparison between numerical and experimental data by generating accurate experimental line sources, avoiding the necessity of geometrical spreading correction for 3-D point-source data. The comparison with 2-D and 3-D numerical modelling is based on the Spectral Element Method. The approach shows the relevance of building a line source by sampling several source points, except the boundaries effects on later arrival times. Indeed, the experimental results highlight the amplitude feature and the delay equal to $\pi / 4$ provided by a line source in the same manner than numerical data. In opposite, the 2-D corrections applied on 3-D data showed discrepancies which are higher on experimental data than on numerical ones due to the source wavelet shape and interferences between different arrivals. The experimental results from the approach proposed here show that discrepancies are avoided, especially for the reflected echoes. Concerning the second point aiming to assess the experimental reproducibility of the source, correlation coefficients of recording from a repeated source impact on a homogeneous model are calculated. The quality of the results, that is, higher than 0.98 , allow to calculate a mean source wavelet by inversion of a mean data set. Results obtained on a more realistic model simulating clays on limestones, confirmed the reproducibility of the source impact.

Key words: Body waves; Controlled source seismology; Surface waves and free oscillations; Wave propagation. 


\section{INTRODUCTION}

Since the early developments of seismic imaging methods in the middle of 20th century, several approaches and algorithms have been proposed. The improvements of this last decade have focused on both qualitative imaging techniques such as migration (e.g. Berkhout et al. 2012; Guofeng et al. 2013), novel applications of quantitative imaging methods such as first arrival tomography (e.g. Bohm et al. 2015), and even more recent approaches like Full Waveform Inversion (FWI; e.g. Perez Solano et al. 2014), see Virieux \& Operto (2009) for a review of the last decade). These refinements are proposed at different scales of application, from near-surface civil engineering structures to global crustal imaging, and include oil prospection. Validation is generally done using well-known shared benchmarks like the Marmousi model (Martin et al. 2006). However, the synthetic data are generally computed using the same wave propagation modelling engine used in the inverse problem process. This approach is particularly useful for validating an algorithm in its early development stage but does not take into account the artefacts associated with the assumptions of the forward problem. On the other hand, it is often challenging to assess the accuracy of a given method on the basis of real experiments, in which precise information on the Earth's interior is absent and possibly leads to geological misinterpretation (Morozov 2004). In this context, controlled experimental measurements appear to be a powerful intermediate step, provided they are capable of producing accurate data.

Small-scale physical modelling methods have been used for several decades to study the propagation of waves in media presenting different levels of complexity, ranging from acoustic wave propagation in homogeneous media to elastic wave propagation in 3-D heterogeneous anisotropic media. This experimental approach was first used to describe the phenomenology of propagating waves (for example Rieber 1936; Howes et al. 1953; Oliver et al. 1954; Angona 1960; O'Brien \& Symes 1971). Small-scale physical modelling was then used to test imaging processes (e.g. Hilterman 1970; French 1974; Bishop et al. 1985; Pratt 1999; Mo et al. 2015), and validate numerical tools (Favretto-Cristini et al. 2014). The technology used for these different works has become increasingly sophisticated (see Bretaudeau et al. (2011) or Valensi et al. (2015) for a more detailed review). Nowadays, most experimental benches include piezoelectric transducers to simulate multisources and multireceivers (Wong et al. 2009) or immersed zero-offset profiles (Favretto-Cristini et al. 2014). Laser interferometry is a recent alternative, providing seismic records free of coupling effects in solid media (Bodet et al. 2005; Van Wijk 2005; Bretaudeau et al. 2011, 2013) and in gel (De Cacqueray et al. 2011). All the above studies have shown the relevance of experimental seismic data obtained under controlled conditions. However, key points need to be addressed in order to quantitatively simulate seismic surface measurements generated with a hammer fall source: first, modelling surface waves prevents the use of immersed media (in which case it would be interface waves), and second, the omni-directionality of the radiation pattern of $P$-waves implies a physical source point. To this end, the Mesures Ultrasonores Sans Contact (MUSC) system has been designed (Bretaudeau et al. 2011) to simulate: (1) wide-angle on-shore acquisitions, modelling both body waves and surface waves; (2) automatic multisource-multireceiver measurements with high-productivity, (3) high-precision source-receiver positioning; and (4) high-precision recording of absolute surface displacement without coupling effects. This type of experimental seismic modelling setup can become a great tool providing a value- added stage in the imaging process validation if the experimental measurement chain is perfectly mastered. This condition is fully met if (1) the experimental data are perfectly reproducible with a numerical tool, as well as if (2) the effective source is reproducible along the measurement setup. However, experimental works above cited, which use piezoelectric sources and a laser interferometer, do not propose any very fine quantitative analysis of numerical comparison of the data nor effective source shape reproducibility. For this reason these two aspects correspond to the objectives of the two studies presented here. The results will define the capacity of using a set of data carried out in MUSC as a universal reference usable for any imaging process by different geophysics research teams.

In the case of MUSC data, the abilities of the system have been first validated experimentally on a small-scale model containing a cavity (Bretaudeau et al. 2011). The comparison with 2-D numerical modelling showed close similarities on the diffracted and converted arrivals, after accounting for the experimental source waveform. However, since the numerical source was simulated in 2-D, some corrections were required to compare the resulting amplitudes, leaving moderate discrepancies that are discussed in Bretaudeau et al. (2011).

In order to quantitatively tackle this issue, we suggest in a first study to continue further the approach through two progressive stages: first of all we test a recent 2-D-3-D correction that allows the comparison between the 2-D-numerical and 3-D-experimental data, then we propose to compare 2-D numerical data with experimental 2-D data provided by a sampled line source. This last process makes possible to avoid any correction of the experimental data before the comparison. Indeed, although real media are rarely 2-D, so far, 3-D elastic wave propagation modelling methods are computationally expensive (Etienne et al. 2010; Borisov et al. 2013; Brossier et al. 2013; Butzer et al. 2013; Borisov \& Singh 2015) and imaging methods are used mainly for 2-D structures (Brossier et al. 2009; Romdhane et al. 2011; Bretaudeau et al. 2013; Groos et al. 2014). Added to the need due to the computational cost of 3-D modelling, the natural process of numerical developments needs gradual stages from 1D to 3-D, including the 2-D case (see e.g. Capdeville et al. 2010). Therefore the majority of forward problems are processed in the 2-D case and implicitly use line sources in 2-D-space while field and experimental data are acquired using punctual sources that provide 3-D wavefields. Thus, in order to adapt the experimental results to 2-D numerical data, a widely used method consists in spreading transformations of 3-D-wavefields for 2-D-media as a pre-processing step (Crase et al. 1990; Shipp \& Singh 2002; Ravaut et al. 2004; Wang \& Rao 2009; Bretaudeau et al. 2013). For that, several 3-D-to-2-D transformation techniques have been proposed, each under the assumption that the medium is either 1-D, or 2-D but invariant along the axis perpendicular to the direction of the receiver-profile. Recently, Forbriger et al. (2014) and Schafer et al. (2014) proposed the hybrid method which makes it possible to correct geometrical spreading with good accuracy for both near- and far-field data, as recalled further on, but it is still an approximation and this method is known to fail to retrieve backscattered wavefield. Thus, an ideal way to validate imaging methods is to work with 2-D experimental dataset in controlled environment, that is, generated by a 2-D source. We propose here to generate this kind of data through an alternative path that consists in carrying out measurements from a source-line and making comparisons between numerical and experimental data following correction using the hybrid method.

The second study presented here consists in identifying the reproducibility of the source impact and thus data repeatability and in 


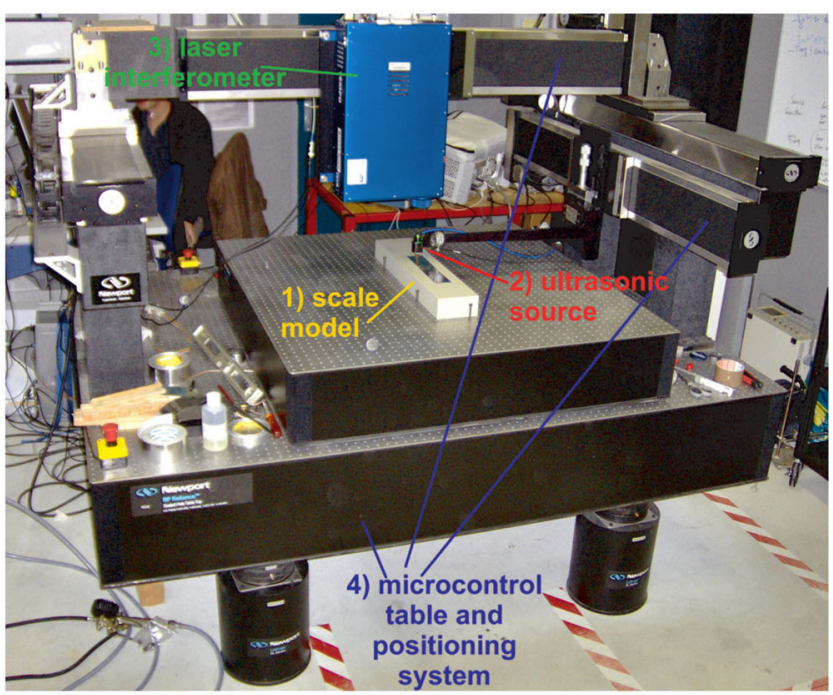

Figure 1. Photograph of the MUSC ultrasonic laboratory (from Bretaudeau et al. 2013) with its four components: (1) a small-scale model of a subsurface zone, (2) a piezoelectric ultrasonic source to generate ultrasonic waves in the model, (3) a laser interferometer to record ultrasonic wave propagation on the model surface and (4) an optical table with two mobile automated arms above the model.

estimating source time functions usable for 2-D imaging methods. The knowledge of the source time function is actually a critical point for high-resolution imaging methods, generally tackled as an unknown parameter in the case of field data. It can be also a critical issue when using a piezoelectric source, as mentioned by (Köhn et al. 2016) who process data with a "Full Waveform Inversion" method and need strategies for overcoming the possible variation of the source coupling impact on the medium surface. In opposite a good approximation of the source excitation allows avoiding source wavelet estimation during the inversion process and facilitating the validation of the imaging method. That is the reason why we propose here to quantify its reproducibility in the measurement bench by comparing results of the inverted source waveforms. This source time function is estimated easily from experimental data, if all the other parameters are mastered, further supporting the use of smallscale physical modelling method for validating seismic imaging methods.

All the experimental tests will be proposed here on realistic models in terms of velocity ratio, attenuation and dispersion of surface waves velocities, in order to study realistic data. The resin models used here for the experimental tests are presented in the first part below with the summary of the specifics of the MUSC laboratory. Afterwards, the numerical characteristics of the code used for providing the simulated data are described before the two last parts where are developed the two coupled studies on experimental data, dealing with the waveform simulation of the propagated signals and the stability of the source wavelet shape.

\section{METHODS}

\subsection{Physical modelling: MUSC laboratory}

The MUSC laboratory (Bretaudeau et al. 2008, 2011, 2013) has been built to experimentally reproduce low noise field seismic data on reduced scale models. Fig. 1 shows the measurement bench and its components: it is composed of a honeycomb tab and two arms that control the source and the receiver positions with a precision of $10 \mu \mathrm{m}$.

The receiving system of the MUSC laboratory is a laser interferometer based on the phase shift of the reflected laser signal due to the particular displacement at the surface of the model during seismic wave propagation in the medium. The diameter of the laser beam on the model surface is $20 \mu \mathrm{m}$ for a focal distance of $40 \mathrm{~mm}$ and makes it possible to detect a vertical displacement of the order of a nanometre in the frequency range of $10 \mathrm{kHz}$ to $20 \mathrm{MHz}$. The laser interferometer constitutes a non-coupled receiver which avoids complicated modelling of coupling effects on measurements.

The seismic source in the MUSC laboratory is simulated by a piezoelectric transducer linked to a launching and synchronization system. It allows choosing the source function, that is, a waveform like a Gauss or Ricker function, for a central frequency $f_{0}$ and a time delay $t_{0}$. To do this, the source is generated by a waveform generator and then amplified before being transmitted to the reduced-scalemodel. The piezoelectric transducers used are built to be adapted to the impedance of the resin model described in the next part. Thus the emitted signal is not resonant but the sensor response combined with the coupling effect to the model behaves as a filter for the source shape, which depends on the frequency. It is possible to assess this effect by taking into account the entire waveform of the pulse as already presented in (Bretaudeau et al. 2011) where this crucial point has been tackled in a first approach with an assumption of 2-D propagation.

In case of small scale modelling, different authors propose an alternative way to only tackle the 2-D phenomena through a 2-D laboratory device which uses guided waves propagation in 2-Dplates (Oliver et al. 1954; Angona 1960; Mo et al. 2015). This principle has several advantages compared to 3-D laboratory: (1) the variety of materials which can be anisotropic and which are less expansive and easier to handle contrary to sometime huge 3-D epoxy-resin models or (2) much less source energy requirement (Oliver et al. 1954; Mo et al. 2015). However, this method is based on the propagation of pseudo-longitudinal waves which are, in fact, non-dispersive Lamb waves while 3-D methods propagate the full wavefield through the model without proxy for propagation velocities and wave types More, 3-D methods are best suited to reflection, refraction and diffraction problems than 2-D methods (Angona 1960) which are critical in high-resolution seismic imaging. In order to avoid these limits, the MUSC laboratory is based on the principle of seismic waves propagation in 3-D blocks.

In this context and for spatial considerations of seismic physical modelling, the coupling part of the piezoelectric transducers must be as close as possible to a point source in order to simulate the spatial energy radiation pattern of a weight drop on the surface, that is, with an omni-directional emitted $P$-wave. Actually, as explained in (Bretaudeau et al. 2011), classical piezoelectrical transducers sizes are generally large compared to the emitted wavelength and provide a directional emission pattern for $P$-waves whereas a hammer fall source used in subsurface field measurements behaves as a punctual impact and provides an omni-directional emission pattern of $P$-waves. For this reason, in the MUSC laboratory, two adapted sources have been tested in term of directivity pattern by (Bretaudeau et al. 2011) who showed their capacity to simulate a thin piston effect. For the spectral band [20 kHz; $200 \mathrm{kHz}$, a commercial piezoelectric transducer is used without any coupling gel. For the spectral band [ $300 \mathrm{kHz} ; 800 \mathrm{kHz}$ ], the piezoelectric source is coupled through a conical adapter stuck to the transducer to obtain the expected impact surface. The resulting radiation patterns of the sources are constantly quasi-omni-directional for $P$-waves in 
Table 1. Physical properties of the two kinds of materials used to build the presented small scale models. $V_{\mathrm{P}}, V_{\mathrm{S}}$ and $V_{\mathrm{R}}$ are the $P$-wave velocity, $S$-wave and the Rayleigh wave velocity, respectively. $\rho$ is the density and $Q$ is the quality factor.

\begin{tabular}{lccccc}
\hline Material & $V_{\mathrm{P}}\left(\mathrm{m} \mathrm{s}^{-1}\right)$ & $V_{\mathrm{S}}\left(\mathrm{m} \mathrm{s}^{-1}\right)$ & $V_{\mathrm{R}}\left(\mathrm{m} \mathrm{s}^{-1}\right)$ & $\rho\left(\mathrm{kg} \mathrm{m}^{-3}\right)$ & $Q$ \\
\hline F50 pure & 2300 & 1030 & 965 & 1300 & 30 \\
LAB1000 & 2850 & 1400 & 1310 & 1500 & 75 \\
\hline
\end{tabular}

the range of the frequencies recorded (see Bretaudeau et al. 2011, for details).

These two frequency bands, called here 'lower frequency band' and 'higher frequency band', have been chosen to allow both complying with the feasibility of machining the heterogeneities in the medium and the dimensional scale ratio. That means : the heterogeneities should be not too small (1 $\mathrm{mm}$ of minimal size) and the total model size should not be too big or too heavy $\left(1 \mathrm{~m}^{2}\right.$ and $270 \mathrm{Kg} \mathrm{max}$ ) for the supporting table. The resulting scale dimensions associated to the propagating parameters of the resins used in this study are presented in the following part.

\subsection{Characteristics of the scale models tested}

In this study, we consider two different reduced scale models. The first is homogeneous whereas the second one, called BiAlt, contains a deeper layer with a geometrical variation of the interface along the profile. The top layer, as well as the entire first model, is made of epoxy-resin called F50 pure. The deeper layer is built with a denser resin called LAB1000. The specific properties of these two kinds of resins are summarized in Table 1.

For the linearized wave equation, the change of scale must conserve the relationship between observables, that is, amplitudes and time arrivals. Regarding the amplitude, the quality factor $Q$ is chosen to be in the same range as the materials of the near surface as shown in Table 1. The key parameter for the time scaling is the ratio between the propagated seismic wavelength and the spatial dimensions of the experiment, which include the model's geometry, the spatial increment between the sources and the receiver positions, as well as the dimensions of the source impact.

For simulating seismic experiments applied to the near surface, we use preferentially the low frequency band described above which allows a dominant wavelength (at $100 \mathrm{kHz}$ ) about $13 \mathrm{~mm}$ for the Rayleigh waves and $28 \mathrm{~mm}$ for the $P$ waves, by taking into account the velocity in the models described in Table 1 . The scale ratio rules used are summarized in Table 2: the scale factor is equal to 2
Table 2. Example of possible scale ratios between field experiments (FEx) and MUSC experiments (MEx) when considering a ratio equal to 1 for the density and quality factors.

\begin{tabular}{lccc}
\hline & FEx scale & MEx scale & Scale ratios \\
\hline$P$-wave velocity & $\mathrm{V}_{\mathrm{p} 0}$ & $\mathrm{~V}_{\mathrm{p} 0}$ & 1 \\
$S$-wave velocity & $\mathrm{V}_{\mathrm{s} 0}$ & $\mathrm{~V}_{\mathrm{s} 0}$ & 1 \\
Time & $\mathrm{T}_{0}$ & $0.001 \mathrm{~T}_{0}$ & 0.001 \\
frequency & $\mathrm{F}_{0}$ & $1000 \mathrm{~F}_{0}$ & 1000 \\
Distance & $\mathrm{D}_{0}$ & $0.001 \mathrm{D}_{0}$ & 0.001 \\
Wavelength & $\mathrm{D}_{0}$ & $0.001 \mathrm{D}_{0}$ & 0.001 \\
\hline
\end{tabular}

between real and reduced model velocities. Thus a central frequency of $100 \mathrm{kHz}$ in the laboratory corresponds to a central frequency of $50 \mathrm{HZ}$ in the field, which is realistic for simulating a hammer impact on a surface. In this case, the distance scale ratio is 1000 , so a distance of $1 \mathrm{~mm}$ in the laboratory experiment corresponds to a distance of $1 \mathrm{~m}$ in reality.

As mentioned before, the laser beam diameter used for recording the propagating waves is $20 \mu \mathrm{m}$ wide. In the presented study, considering the dominant wavelength (at $100 \mathrm{kHz}$ ) equals about $13 \mathrm{~mm}$ for the Rayleigh waves and $28 \mathrm{~mm}$ for the $P$ waves, the laser beam respectively equals $\lambda / 650$ and $\lambda / 1400$. At the field scale, and following the rules in Table 2, those correspond to measurement surfaces of $20 \mathrm{~mm}$ and $2 \mathrm{~mm}$ respectively. These dimensions are lower or equivalent to the possibilities available by geophones holds. Thus, at the laboratory scale in MUSC, the measurement accuracy in term of the size of the recording surface is respected.

In parallel, the position accuracy of the receiving system is $10 \mu \mathrm{m}$. This value is lower to the focal beam. It corresponds to the centre position of the laser beam, which is $20 \mu \mathrm{m}$ large. In practice, the incremental displacement between two receivers positions used generally equals $1 \mathrm{~mm}$. It is higher than the focal beam size.

Furthermore, as shown on Fig. 2, even if the emission properties of a piezoelectric transducer is narrow-band, the spectral bandwidth of the pulse emitted by the piezoelectric transducer is large enough to simulate a seismic pulse emitted by a hammer fall in subsurface media, through the scale ratio used in Table 2: the frequency of $100 \mathrm{kHz}$ in MUSC corresponds to $50 \mathrm{kHz}$ at field scale and $150 \mathrm{kHz}$ corresponds to $75 \mathrm{~Hz}$.

In the next section, the recorded signals will be analysed for a maximum offset equal to $60 \mathrm{~mm}$ in the case of the homogeneous model and $100 \mathrm{~mm}$ for the BiAlt model. Thus the resin models have to be wide enough to accommodate this receiver-source distance without creating boundary echoes that could interfere with direct arrivals. To do this, the homogeneous model is $500 \mathrm{~mm}$ long, (a)

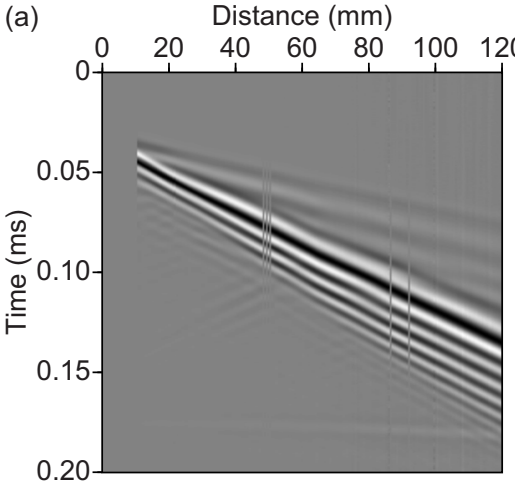

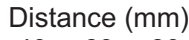

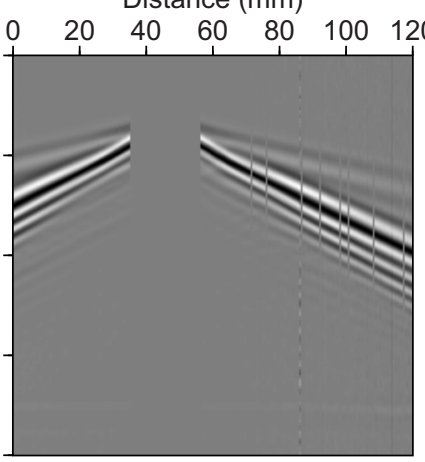

Distance $(\mathrm{mm})$

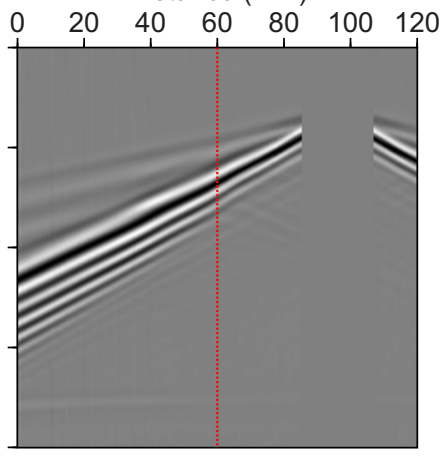

(b) Norm. Amp.

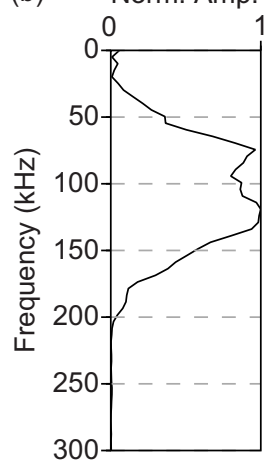

Figure 2. (a) Examples of multireceiver records in the MUSC laboratory for a two-layer model (BiAlt model). Zero-value data correspond to the diameter of the source. (b) Normalized amplitude spectrum of a recorded trace (red line in (a)). 
(a)
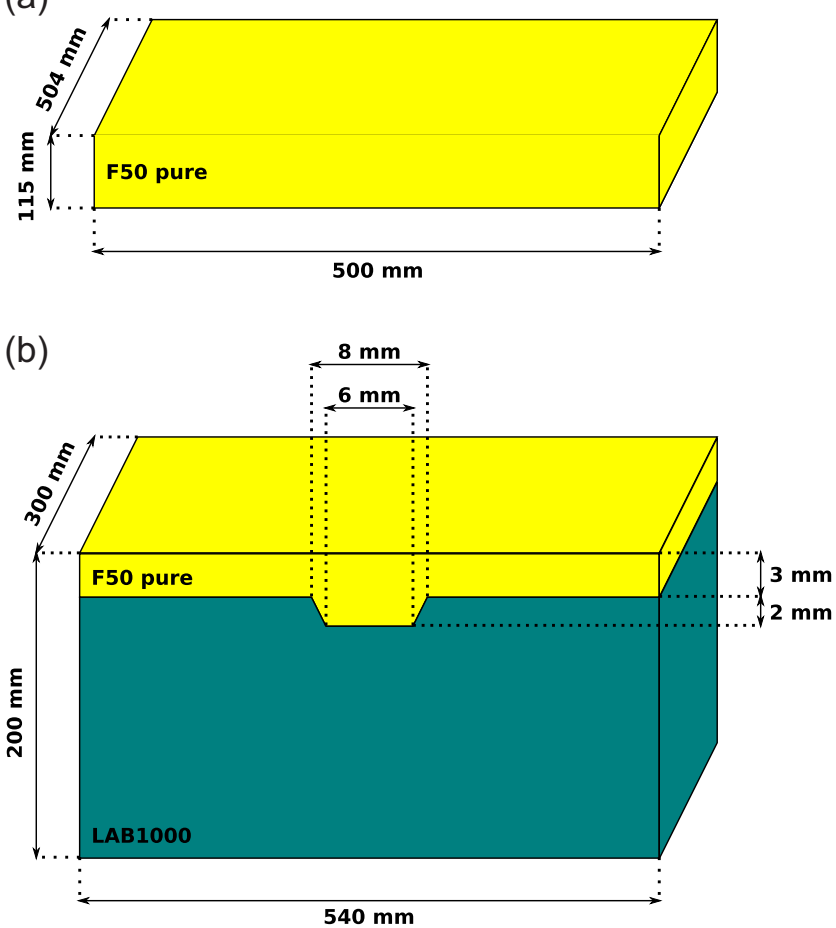

Figure 3. Schematic representation of (a) the homogeneous model used in this study and (b) the BiAlt model.

$504 \mathrm{~mm}$ large and $115 \mathrm{~mm}$ high. The BiAlt model is $540 \mathrm{~mm}$ long, $300 \mathrm{~mm}$ large and $203 \mathrm{~mm}$ high. The geometry is presented in Fig. 3 and simulates an interface between a $3 \mathrm{~m}$ thick layer of clay over a limestone layer. Thus, the BiAlt model can be seen as a realistic model providing realistic data.

These two resin blocks and their corresponding numerical models will be used for generating seismic data with punctual sources and line sources for the homogeneous model, in order to study the effective source excitation emitted on the MUSC bench and its reproducibility.

\subsection{Numerical modelling: Spectral Element Method}

For this study we need a numerical modelling method whose spatial discretization is suitable for representing complex environments and which provides both high precision results and low numerical dispersion. Thus we use the Spectral Element Method (SEM) for 2-D and 3-D elastic wave propagation modelling (Komatitsch \& Vilotte 1998; Komatitsch et al. 1999, 2005; Festa \& Vilotte 2005).

SEM is a variant of the Finite Element Method (FEM) (Lysmer \& Drake 1972; Seron et al. 1990; Hulbert \& Hughes 1990; Tromp et al. 2008) based on a high-order piecewise polynomial approximation of the weak formulation of the wave equation which leads to a spectral convergence ratio as the interpolation order increases. Considering near-surface experiments, one advantage of SEM is that the weak formulation naturally satisfies the free-surface condition used to simulate surface wave propagation with considerable accuracy (Komatitsch \& Vilotte 1998; Komatitsch et al. 1999, 2005). Contrary to FEM, which calls on a wide range of available element geometry (Dhatt \& Touzot 1984), SEM is limited to quadrilateral elements in 2-D and hexahedral elements in 3-D. Note that although SEM with tetrahedral elements exists (Komatitsch et al. 2001) it leads to theoretical complications. However, quadrangles and hexahedras are well suited for handling complex geometries and interface matching conditions (Cristini \& Komatitsch 2012).

In SEM, the wave-field is expressed in terms of high-degree Lagrange interpolants and the calculations of integrals are based on the quadrature of Gauss-Lobatto-Legendre (GLL). Each element is discretized with Lagrange polynomials of degree $n_{l}$ and contains $n_{l}+1$ GLL points that form its local mesh. This combination of high-degree Lagrange interpolants with the GLL integration leads to a perfectly diagonal mass matrix which in turn provides a fully explicit time scheme suitable for numerical simulations on parallel computers (Komatitsch \& Vilotte 1998; Komatitsch et al. 1999).

The spatial resolution of SEM is controlled by the typical size of an element $(\Delta h)$ and the polynomial degree in use on an element $\left(n_{l}\right)$. Typically, a polynomial degree $n_{l}=4$ is optimal for seismic wave propagation modelling (Moczo et al. 2011) although $n_{l}=8$ remains numerically affordable in 2-D. To obtain accurate results, the required $\Delta h$ is of the order of $\lambda_{\min } / 2<\Delta h<\lambda_{\min }$ for $n_{l}=4$ and $\lambda_{\text {min }}<\Delta h<2 \lambda_{\text {min }}$ for $n_{l}=8, \lambda_{\text {min }}$ being the smallest wavelength of the waves propagated in the model. The time marching scheme is governed by the CFL stability condition:

$\Delta t<\mathcal{C} \frac{\Delta h}{c_{\max }}$

where $\mathcal{C}$ is the Courant constant and $c_{\max }$ is the maximum wave velocity, typically the $P$-wave velocity. The Courant constant $\mathcal{C}$ is determined empirically, depending on the application, and is fixed at a maximum of 0.30 for this study.

The numerical meshing required for the numerical simulations involves cell dimensions of about $e_{s}<3.43 \mathrm{~mm}$ for the F50 material and $e_{s}<4.66 \mathrm{~mm}$ for the LAB1000 material, considering a polynomial degree $n_{l}=4$ and a slightly over-estimated maximum frequency of $f_{\max }=300 \mathrm{kHz}$ for $f_{0}=100 \mathrm{kHz}$. In our study, the models are meshed with quadrangles (2-D) and hexahedra (3-D) using the GMSH open-source software package (Geuzaine \& Remacle 2009).

\section{FROM POINT-SOURCE TO LINE-SOURCE RESPONSE}

The approach described here consists in generating experimental data with a line source as well as a 3-D point source and analysing the similarity with numerical results under the same conditions. This is done to respond to two needs: (1) the quantitative refined validation of the reduced scale data, (2) the capacity of the reduced scale bench to generate 2-D data sets that are intermediate between numerical simulation and field data suitable for the 2-D imagery tests. Indeed, in the framework of wave propagation modelling and imaging methods, although 3-D acoustic algorithms exists (BenHadj-Ali et al. 2008; Plessix et al. 2010) and 3-D elastic algorithms are still being developed (Castellanos et al. 2011; Borisov \& Singh 2015), most available algorithms are limited to 2-D elastic and 3-D acoustic approximation, mostly due to reasons of computational cost. Furthermore, validation of the inversion process is often limited to processing made with synthetic data using the same model for computing both predicted and estimated measurement, while the validity of applications on real datasets is conditioned by good a priori and poor knowledge of the target. All of these lead to a limited validation of the efficiency of imaging methods to recover parameter models. Thus accurately correcting the difference between 2-D and 3-D geometrical spreading is critical for the 2-D inversion of field data. 


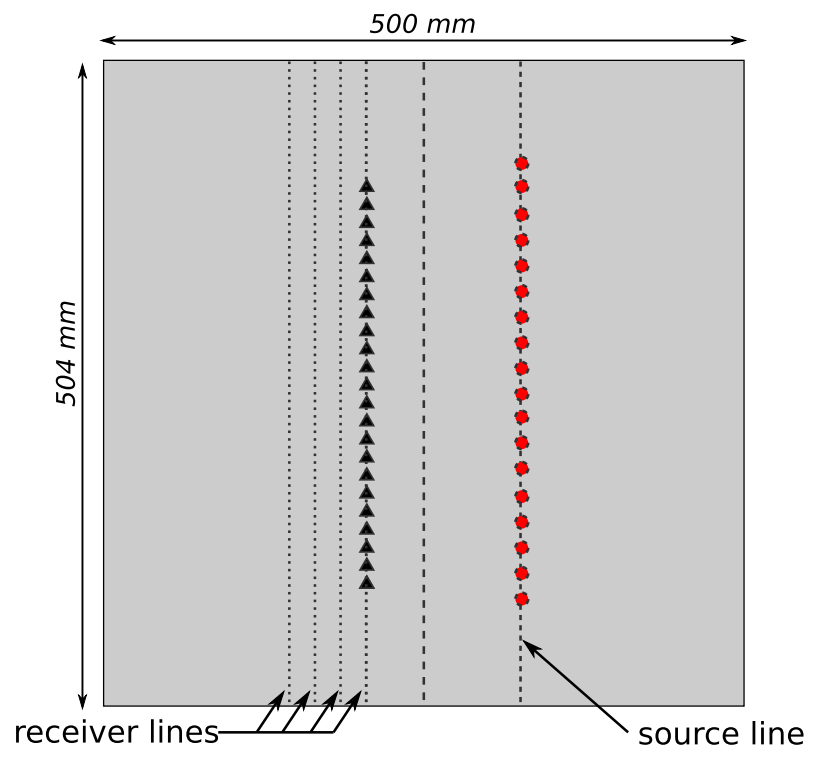

Figure 4. Schematic representation of the acquisition geometry used to generate an experimental line source, that is, an equivalent of cylindrical source use in 2-D modelling. The black triangles and red circles represent receivers and sources, respectively.

Many strategies exist to correct geometrical spreading effect differences between 3-D and 2-D data, the two widely used are (1) data convolution with $\sqrt{t^{-1}}$ together with the application of a $\sqrt{t}$ taper function (Crase et al. 1990; Shipp \& Singh 2002; Ravaut et al. 2004) and (2) data tapering $(\sqrt{t})$ and inference of a source function by linear inversion to estimate the correct the phase (Pratt 1999; Bretaudeau et al. 2013). However, these strategies are used to transform data which contains essentially body waves (except Bretaudeau et al. 2013) and the first one is known to produce artefacts which can be significant (Auer et al. 2013). To overpass these limitation and transform near-surface data dominated by surface wave, Forbriger et al. (2014) have recently developed the hybrid method and validate it through numerical tests (Schafer et al. 2014).

The hybrid method summarized in the Appendix is an efficient spreading transform which makes it possible to reconstruct velocity models with the 2-D-FWI method using 3-D-data. However, this method is derived from a far-field acoustic approximation of Green's functions and is known to fail for back-scattered surface waves (Schafer et al. 2014; Groos et al. 2014). Moreover, the correct smooth transition between so-called single-velocity and directwave transformations, which are the two components of the hybrid method, is not easy to determine without 2-D reference data. This is the case for all experimental data and the spreading transformation results become strongly dependent on the user's attempts, expertise and know-how. Thus the missing step between purely numerical validation and real data applications should be addressed by an alternative approach that consists in recording experimental seismograms generated by line sources under controlled conditions. Here, we take advantage of the experimental framework to explore this alternative approach specific to the MUSC laboratory, that is, by carrying out 2-D measurements from 3-D constructed line source. Fig. 4 shows a schematic representation of the principle of this kind of experiment. The line source is composed of a finely sampled line of point sources and a line of receivers for each offset considered, as explained below.

For practical reasons in the MUSC laboratory, the experiment is carried out through the principle of reciprocity in the case of recording a vertical source and a vertical component, with one source position and a set of receiver positions spread out along the invariant direction. For each source position (i.e. for each offset), all traces of each set of receivers are then stacked together to obtain the 3-D constructed line source response. Note that 3-D constructed line-source response corresponds to the wavefield generate by a line source in a 3-D space. In order to apply this protocol, we choose a suitable sampling interval $\Delta s$ between each point source constituting the 3-D constructed line source to ensure the applicability of Huygens principle. Given the material properties of F50 pure epoxy-resin, we choose an interval $\Delta s=\lambda_{\min } / 10 \cong 0.5 \mathrm{~mm}$ over a line of $300 \mathrm{~mm}$ long which leads to 601 point-source positions.

Four receiver positions are available: $45,50,55$ and $60 \mathrm{~mm}$ offset. The source time function (for the numerical simulation as well as for the experimental test) is a Ricker wavelet, the second derivative of a Gaussian function:

$s(t)=\left(1-2\left(\pi f_{0}\left(t-t_{0}\right)\right)^{2}\right) e^{-\left(\pi f_{0}\left(t-t_{0}\right)\right)^{2}}$,

where $f_{0}$ is the central frequency and $t_{0}$ is the peak time. Here, we take a central frequency $f_{0}=100 \mathrm{kHz}$ and $t_{0}=0.03 \mathrm{~ms}$. The data sets obtained are filtered using a low-pass Butterworth filter with a cut-off frequency $\omega_{c}=250 \mathrm{kHz}$ to remove noise and tapered at the beginning using a cosine taper function of width $w=0.03 \mathrm{~ms}$ on the time signal. The 3-D constructed line-source data is obtained through a weighted stack over the common offset traces. Fig. 5 shows the results for both the numerical simulation and the experimental data. The signals emitted by a line of point sources and recorded at the first receiver position $(45 \mathrm{~mm})$ are presented in Figs 5(a) and (c) for the numerical and experimental tests, respectively. Note that no attenuation is accounted for in the numerical modelling, so we do not compare, in Fig. 5, the numerical and experimental results directly. Moreover, all the resulting traces are normalized to be comparable to the experimental tests. The numerical result (Fig. 5a) clearly shows the attempted direct $P$ - and Rayleigh (non-dispersive Rayleigh in homogeneous media) wave fronts and the reflected $P P$ and $P-S V$ wave fronts, as mentioned previously (labels 1, 2, 3, 4 in Fig. 5). These similarities between the numerical simulation and the experimental data are altered by multiple echoes visible on the experimental data (labelled E in Fig. 5b), as a ringing effect on the source wavelet due to the piezoelectric transducer coupling on the model surface. This point will be addressed in the next section which focuses on source reproducibility.

Figs 5(b) and (d) present the comparisons of 3-D point-source (red line) and 3-D constructed line-source (green line) data at the first receiver position (45 $\mathrm{mm}$ offset) for the numerical and experimental tests, respectively. To complete the comparison, a 2-D modelling result is added in Fig. 5(b) (bold grey line) as a reference.

Fig. 5(b) shows that the 3-D constructed line-source data (green line) provided with a set of punctual sources is perfectly superimposed on the 2-D reference data (bold grey line) until $0.18 \mathrm{~ms}$. Afterwards, the $P-S V$ amplitude (i.e. the last arrival) is abnormally high for the sampled line-source data. As shown in eq. (A6), a line source is a sum of an infinite number of point sources along an axis. Here, the finite dimensions in space of the experimental 3-D setup can cause boundary effects and thus this difference in amplitude.

For the earlier arrival, the global fit between the numerical data highlights the validity of sampling a finite line source by a set of point sources. Concerning both the numerical (Fig. 5b) and experimental (Fig. 5c) cases, the comparison of 3-D point-source and 3-D constructed line-source data shows the expected phase shift of $\pi / 4$ (see eq. A7). Similar comparisons for the four source-receiver 

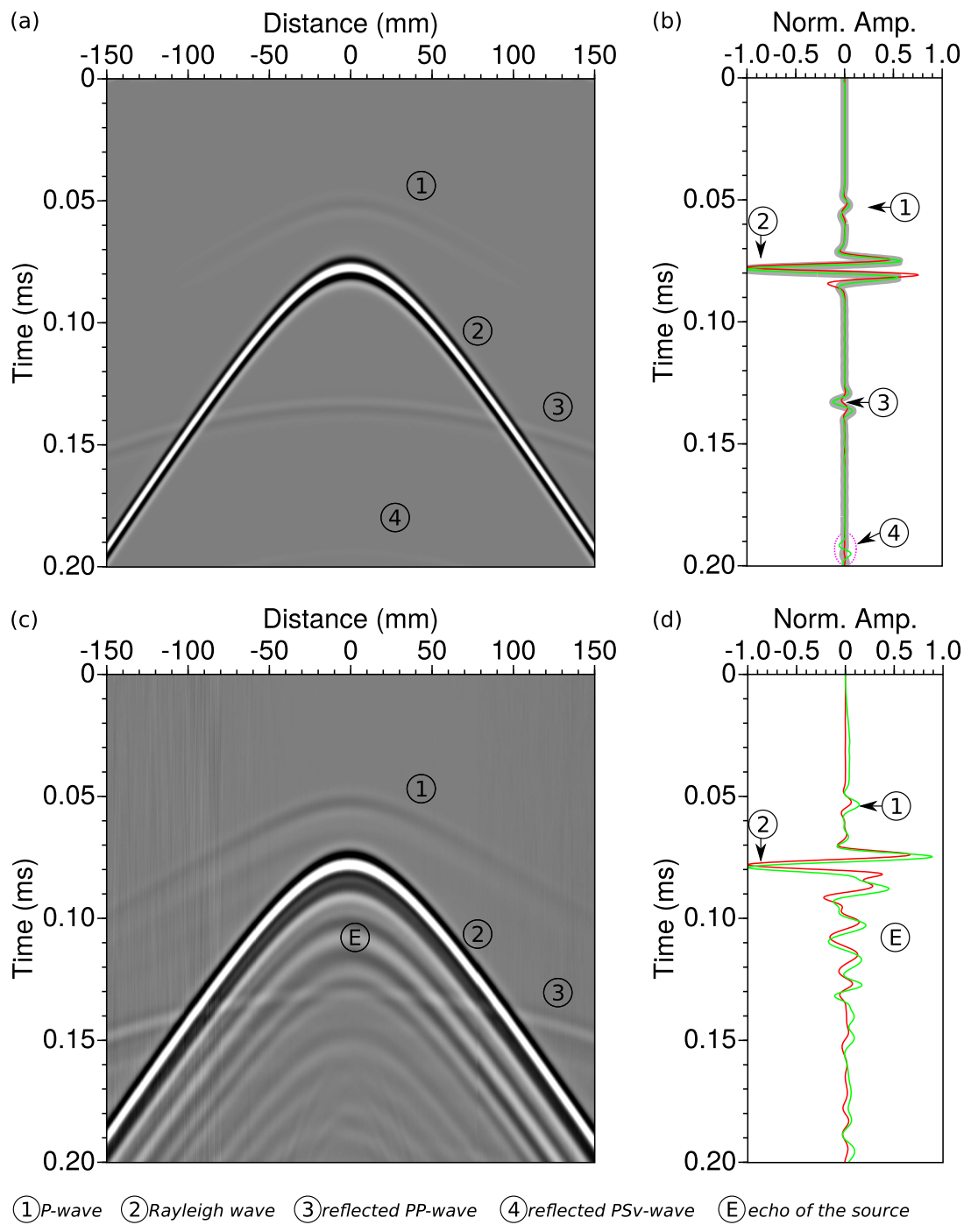

Figure 5. (a,b) Numerical modelling. (a) The resulting seismogram at one receiver position for the experimental line source. (b) Comparison between 3-D point-source response in red (central trace of (a)), 3-D constructed line-source response of (a) in green and 2-D line-source response from 2-D modelling in grey. (c,d) The same as (a) and (b) but for experimental modelling.

offsets are shown in Figs 6(a) and (c) for numerical and experimental data, respectively.

To test the improvement of our approach to provide line-source data in comparison to spreading transformation methods, our results are compared to data obtained using the hybrid method. Figs 6(b) and (d) present the comparison between the 3-D constructed linesource data acquired from a set of punctual sources (green line) and transformed 3-D point-source data (blue line). The comparison of numerical results (Fig. 6b) shows that the hybrid method is able to produce equivalent line-source data with very good agreement in terms of both amplitude and phase for direct arrivals. However, $P P$ and $P-S V$ reflected waves (back-scattered wave) remain different. The same comparison is made for the experimental data in Fig. 6(d). This last result also shows good agreement between line-source and transformed point-source data up to $0.12 \mathrm{~ms}$ (mainly directwaves). However, discrepancies occur for the reflected waves. The first reflected wave is marked by a red line in Figs 6(b) and (d).

These disagreements are more marked than in the numerical case: the correction of the geometrical spreading with the hybrid method seems unable to correctly scale the amplitude due to interference from the echoes of the source and reflected wave. Consequently, a 3-D constructed line source should be recommended instead of the hybrid correction of data to take into account all the seismic arrivals. Concerning the signal recorded at the $55 \mathrm{~mm}$ offset, the largest difference in amplitude can be explained by a weaker signalto-noise ratio than for the three other offsets in the experimental data.

These results obtained using our approach to generate 3-D experimentally constructed line-source data show that the MUSC laboratory is efficient and can produce reliable experimental 2-D data suitable for migration-based methods such as 2-D-FWI. Thus it plays the role of an intermediate tool capable of providing 3-D point-source and 3-D constructed line-source data without the need for geometrical spreading corrections.

\section{EXPERIMENTAL SOURCE TIME FUNCTION}

In the framework of high-resolution imaging, such as FWI, the first validations of the method are generally performed with synthetic 

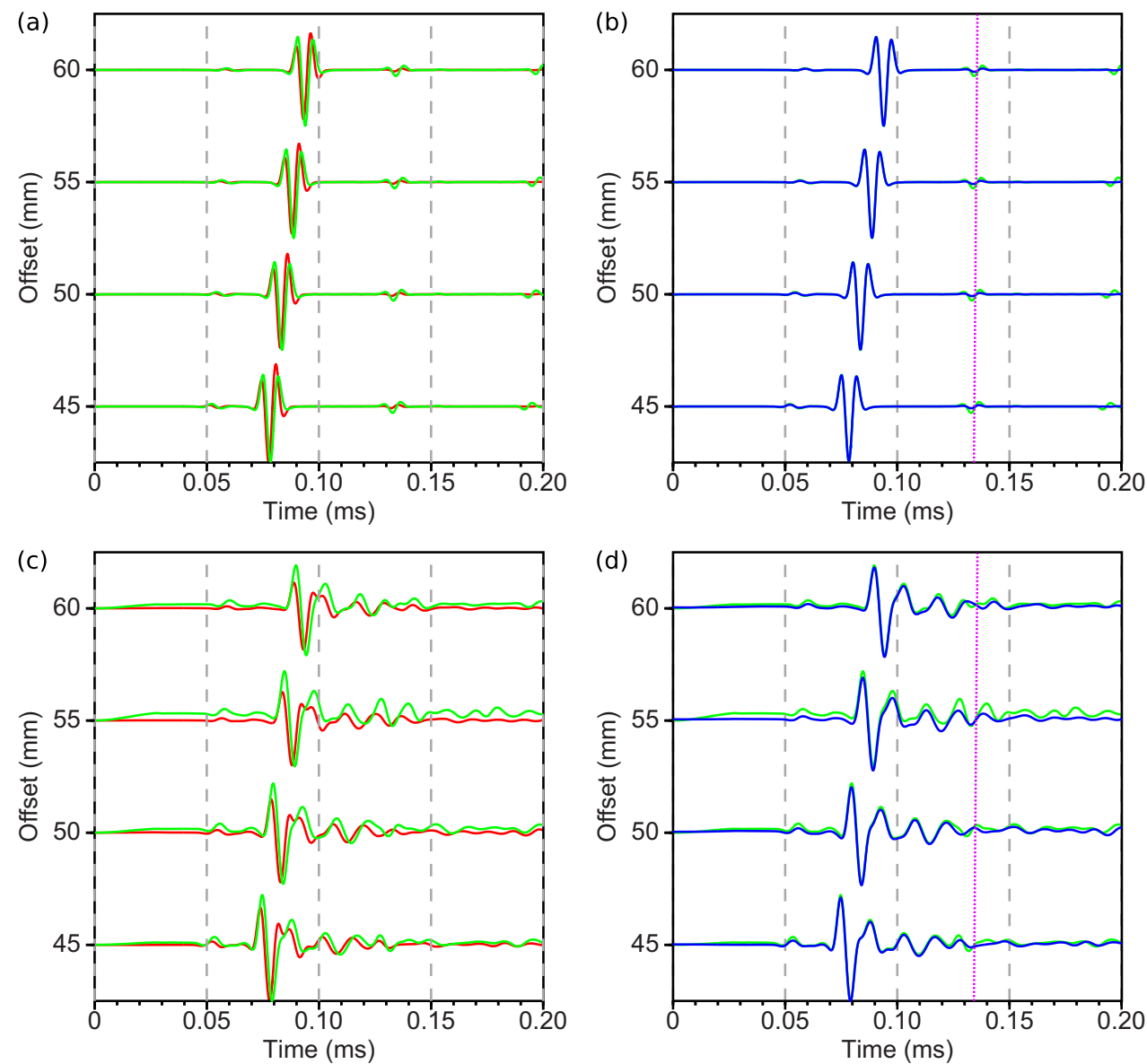

Figure 6. Numerical model. (a) Comparison between synthetic seismograms for a 3-D point source (red) and for a 2-D line source (green), for 45, 50, 55 and $60 \mathrm{~mm}$ source-receiver offsets respectively. (b) Comparison between synthetic seismograms for a 2-D line source (green), and a 3-D point-source response corrected from geometrical spreading (blue) for the same source-receiver offsets as (a) using the hybrid method with ratios $r=0.35, r=0.40, r=0.45$ and $r=0.50$ for offsets $45,50,55$ and $60 \mathrm{~mm}$, respectively. (c,d) the same as (a) and (b) for experimental modelling. The light-purple dotted lines depict the peak $P-S V$-wave front.

data generated with the same modelling engine than the one used for inversion. In these cases, the source waveform is known and the initial model $m_{0}$ is generally a smoothed version of a known true model used in the forward problem to obtain synthetic observed data. Consequently, no source wavelet estimation is necessary. However, obtaining knowledge of the original source time function is an important task when real data are inverted. The solution of the source is obtained using a linear source wavelet estimation (Pratt 1999; Virieux \& Operto 2009) which can integrate all the data from multisource/multireceiver acquisition and the estimated source excitation is given by

$S_{\mathrm{est}}(\omega)=\sum_{i=1}^{N_{S}} \sum_{j=1}^{N_{R}} \frac{H_{i j}(\omega) G_{i j}(\omega)^{*}}{H_{i j}(\omega) H_{i j}(\omega)^{*}} S(\omega)$,

where $\omega$ is the angular frequency, $S_{\text {est }}$ is the real Fourier transform of the estimated source, $G(\omega)$ is the real Fourier transform of the observed signal, $H(\omega)$ is the real Fourier transform of the signal calculated in the synthetic model, $S(\omega)$ is the synthetic source used to compute $H(\omega), N_{S}$ is the number of sources, $N_{R}$ is the number of receivers and ${ }^{*}$ denotes the conjugate. The main issue of this method is that inaccuracies in the synthetic model, and consequently in the calculated data, are integrated in the estimated source. The resulting distortion of the estimated source wavelet can lead to inaccuracies in the updated models during data inversion and then in the recovered parameters of the final model. Moreover, for a given data set, one or more specific sources need to be estimated, depending on whether the source is considered stable enough from one shot to another or not. However, estimating the source for each shot in the case of numerous multisource/multireceiver data can lead to significant additional numerical cost. Thus knowledge of the source time function and its stability are two crucial key points in modelling experimental data for testing imaging processes.

We showed in the previous section that the MUSC laboratory is able to generate high quality experimental 3-D constructed linesource seismograms. If the source waveform is constant during a multisource/multireceiver experiment, it will be very efficient for validating the imaging method. As shown by Bretaudeau et al. (2011), the source waveform injected into the reduced-scale model by the piezoelectric source is different from the selected theoretical one. Indeed, Figs 5(c) and (d) show multiple wave fronts following that of the first arrival. These multiple echoes are due to the coupling of the piezoelectric source on the material. This can depend on the material as well as the force applied on the transducer and naturally raises the question of the ability of the MUSC laboratory to provide reproducible sources during a complete multisource/multireceiver experiment. In order to evaluate the reproducibility of the source impact, several numerical and physical models described below 


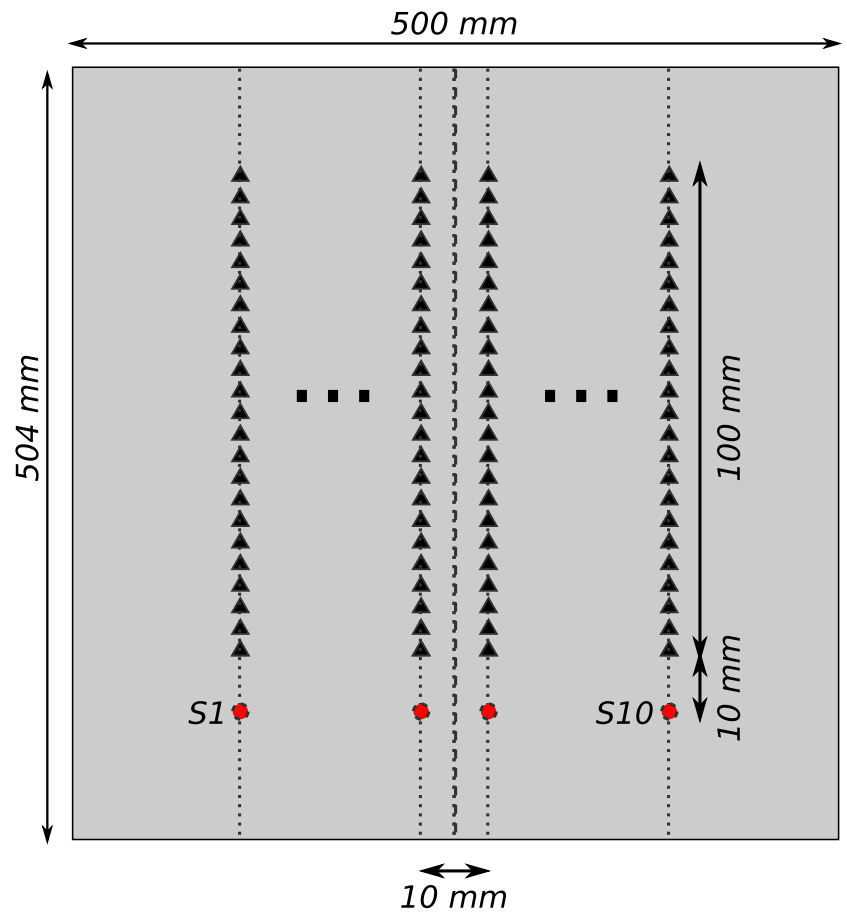

Figure 7. Schematic representation of the acquisition geometry used to assess data reproducibility using the MUSC laboratory. The black triangles and red circles represent receivers and sources, respectively.

were applied to the same $F 50$ pure homogeneous epoxy-resin block as in the previous section.

In the first step, ten events were acquired for this model with a similar geometrical setup: 120 receiver positions with an increment of $\Delta r=1 \mathrm{~mm}$ and a minimum source-receiver offset of $r=10 \mathrm{~mm}$ (see Fig. 7). The numerical wavelet sent to the piezoelectric transducer source is a Ricker function with a central frequency of $100 \mathrm{kHz}$ and $t_{0}=0.03 \mathrm{~ms}$. Each data set is filtered using a low-pass Butterworth filter with a cut-off frequency of $\omega_{c}=250 \mathrm{kHz}$ to remove noise and tapered at the beginning using a cosine taper function of width $w=0.03 \mathrm{~ms}$. Then, a 3-D/2-D geometrical spreading correction is applied using the hybrid method. As shown previously, this correction is well adapted for correcting direct arrivals which are preferentially taken into account to determine the source wavelet. Fig. 8 shows the resulting central trace $(r=70 \mathrm{~mm})$ of each realization (red line signals) compared to a reference central trace resulting from the average of traces for the same offset (signal indicated by a green line). The good agreement between the central traces and the reference signal is the first validation of the reproducibility of the source in the same experiment. This agreement is strengthened by a correlation coefficient higher than 0.98 in each case. To go further in this comparison, a mean correlation coefficient, for all the traces, is calculated for $P$-, Rayleigh and $P P$-waves. The mean correlation coefficients for Rayleigh and $P P$-waves are close to the maximum (0.99) and confirm the good agreement between traces for these phases. For the $P$-wave, the mean correlation coefficient is smaller (0.84) but still good. This last can be relied to the signal-to-noise ratio which can vary from an experiment to another and impact mostly the $P$-wave as it can be seen on shot 10 (Fig. 8).

In the second step, a unique source wavelet is estimated using eq. (3). As done previously, the signals are normalized to avoid the intrinsic attenuation effects on the direct arrivals. The source wavelet estimation takes into account the vertical components of the ten experiments together and allows obtaining a mean effective

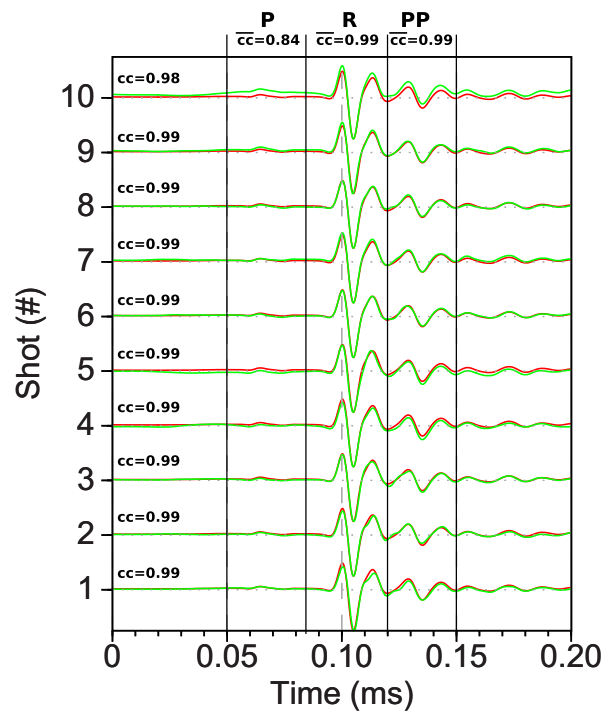

Figure 8. Central trace for each of the 10 analogical experiments compared to a mean central trace (green). $c c$ gives the correlation coefficient between the traces compared for the whole signal while $\overline{c c}$ gives the mean correlation coefficient between the traces compared for a given phase $(P$, Rayleigh, $P P)$.

source time function (Fig. 9). This effective source is very different from the theoretical one, with strong asymmetry around the main peak at $t_{0}$ and a long sequence of source echoes from $t=0.04 \mathrm{~ms}$ to the end of the time window. This source wavelet, convolved with all the synthetic signals, should reproduce the experimental data if the real source wavelet is the same for all the experiments. The resulting traces are presented in Fig. 9(b), which shows that the corrected synthetic seismograms are in good agreement with the experimental ones, with a correlation coefficient higher than 0.92 in each case. These correlation coefficients are not as good as the previous ones. This can be explained first by the fact that the 3-D2-D geometrical spreading correction applied to the experimental traces is not fully efficient for later arrivals, and second because we neglected the effects of quality factors. Consequently, the estimated source is close to the real one but contains the inaccuracies from both the numerical modelling and the geometrical spreading correction. Again, we estimate mean correlation coefficients for $P$-, Rayleigh and $P P$-wave and note the good agreement for Rayleigh and $P P$ waves. However, the mean correlation coefficient for the $P$-wave is quite low (0.64). Indeed, in addition of the signal-to-noise ratio, the potential inaccuracies of the numerical model $(P$-wave and $S$-wave velocity) and the absence of intrinsic attenuation strongly impact the source estimation.

However, these latter results based on an average estimated source wavelet show that the source time function emitted by the transducer in the MUSC laboratory measurement bench is stable enough to ensure robust source reproducibility for a complete physical experiment with multiple source and receiver positions. Therefore, concerning the key issue of source data, the experimental data acquired in the MUSC laboratory can be efficiently processed by imaging methods such as FWI with only one estimation step for all the multisource and multireceiver data.

\section{APPLICATION TO COMPLEX MODEL}

In the previous approaches developed for the geometrical spreading correction calibration and the source estimation, the studies were performed on a homogeneous block of F50 epoxy-resin. This 
(a)

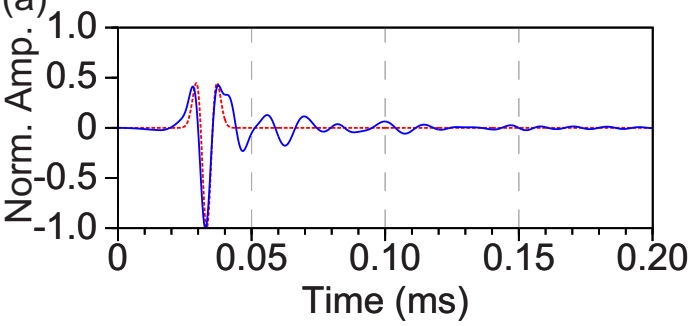

(b)

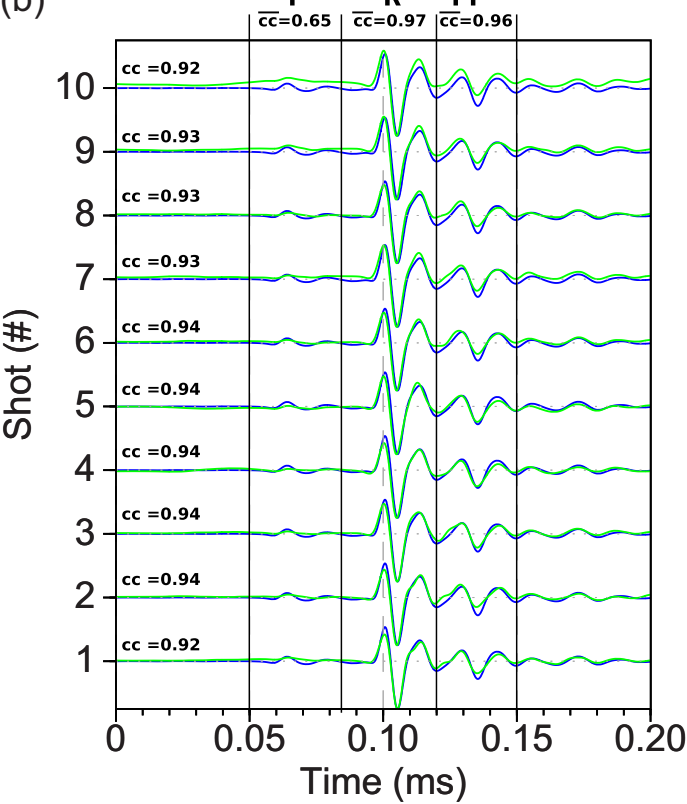

Figure 9. (a) Comparison between the theoretical Ricker source ( $f_{0}=100 \mathrm{kHz}, t_{0}=0.03 \mathrm{~ms}$ ) transmitted to the piezoelectric transducer (dashed red line) and the effective source for the homogeneous F50 pure model (blue line). (b) Comparison between experimental central and numerical traces using the effective source instead of the theoretical one. $c c$ gives the correlation coefficient between the traces compared for the whole signal while $\overline{c c}$ gives the mean correlation coefficient between the traces compared for a given phase $(P$, Rayleigh, $P P)$.

approach facilitates developments and applications but limits the validation to a simple medium with a simple acquisition geometry. Thus here we consider a more complex model called BiAlt (see Section 2.2 for description). The acquisition setup is composed of shots with 241 receivers spaced by $\Delta r=0.5 \mathrm{~mm}$. The receiver line $120 \mathrm{~mm}$ long is centred on the medium axis, where the topography of the two-layer interface provides a valley-shape curve layout for which 25 source positions are considered, ranging from 0 to $241 \mathrm{~mm}$ with a spacing of $\Delta s=10 \mathrm{~mm}$. The source wavelets are modelled by a Ricker function with a central frequency equal to $f_{0}=75 \mathrm{kHz}$ and parameter $t_{0}=0.03 \mathrm{~ms}$. A low-pass Butterworth filter $\left(\omega_{c}=200 \mathrm{kHz}\right)$ and a cosine taper are applied to the data. Given that the top layer of the model is made of the same epoxy-resin as for the homogeneous block, we applied the hybrid geometrical spreading correction with the same parameters. The corresponding synthetic data were generated using a 2-D SEM algorithm. Again, the quality factor is not taken into account. Fig. 10(a) shows the effective source wavelet estimated from the $241 \times 25$ traces compared to the theoretical one. In this case, the estimated source wavelet seems more symmetrical than those recovered for the previous experiment. Moreover, few and very low amplitude multiple
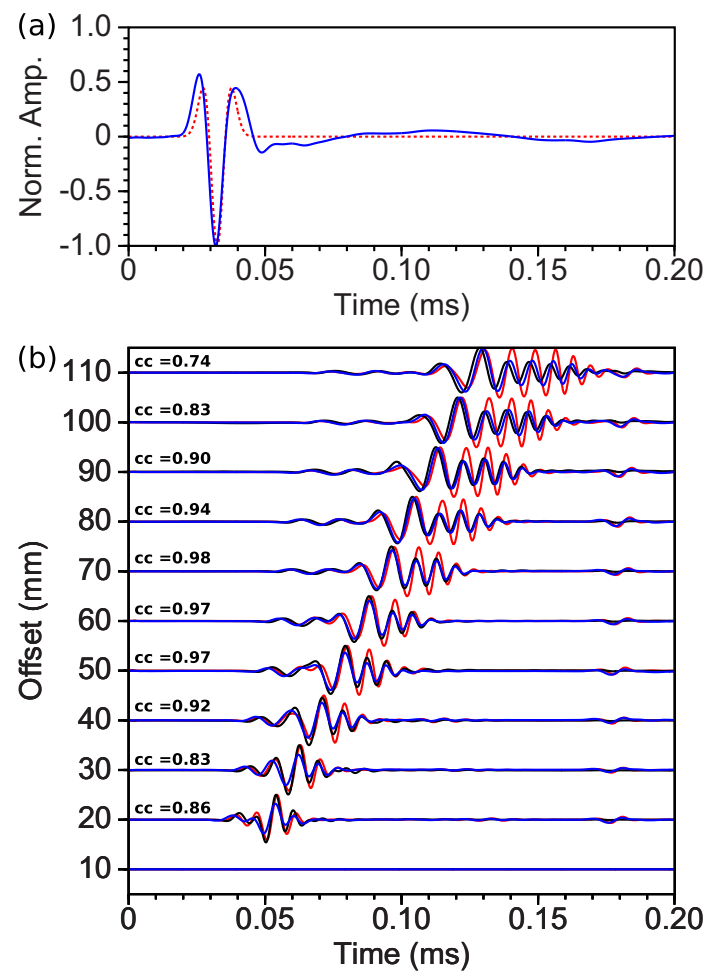

Figure 10. (a) Comparison between the theoretical Ricker source ( $\left.f_{0}=75 \mathrm{kHz}, t_{0}=0.03 \mathrm{~ms}\right)$ transmitted to the piezoelectric transducer (dashed red line) and the effective source for the BiAlt model (blue line). (b) Comparison between experimental central (black) and numerical traces using theoretical source (red) and numerical traces using the effective source (blue). cc gives the correlation coefficient between experimental traces and numerical traces using the effective source.

echoes occurred compared to the previous estimated wavelet. This could be related to the lower central frequency of the source which may generate fewer multiple reflections at the interface between the piezoelectric source and the surface of the material surface. Once again, this estimated source is convolved with the synthetic data and the resulting traces for the first source are shown in Fig. 10(b) (blue line). The comparison between the experimental traces (black) and numerical traces computed with the Ricker source wavelet (red) shows that the relative amplitude between the $P$ and Rayleigh wave fronts are very different, in particular between the intermediate and far offset. The convolution with the estimated source provides good agreement between the experimental data and the numerical data and highlights the relevance of the source inversion for the imaging process. The residual discrepancies were due to the estimation of the quality factor of the epoxy resin $L A B 1000$ that will be refined in further studies. Moreover, given that the effective source is estimated using a realistic multisource/multireceiver design with 25 source positions, these results confirm the stability of the source during large experimental campaigns.

\section{CONCLUSIONS}

High-resolution seismic imaging methods are mostly developed with 2-D approximation and require real data to complete the validation of the inversion process often limited to processing made with synthetic data using the same model for computing both predicted and estimated measurement. We demonstrated here that the geometrical spreading and amplitude corrections usually used to 
transform 3-D in 2-D real seismic data are limited and can be replaced by accurate experimental 2-D data recorded in a controlled environment. This alternative process was shown to be more accurate when taking into account all the arrivals, especially when ringing interfere with the direct arrivals.

In the second step, the effective source wavelet emitted in the material after the coupling effect of the transducer and its possible variability were studied. Given that knowledge of the source is an important aspect for certain seismic data inversion algorithms, source estimation is performed using the linear source wavelet estimation method which integrates the entire signal and is strongly dependent on the accuracy of the initial numerical model. It is preferable to have the same source wavelet throughout a complete experiment. Thus we studied the experimental source and validated its good reproducibility for multisource/multireceiver experiments in the case of a homogeneous medium and for a two-layer model having an internal interface with varied topography. The good repeatability of the source wavelet recovered and the high correlation coefficient of the simulated data in comparison to the experimental data, demonstrated the quality of the experimental data obtained using the MUSC reduced scale measurement bench. Thus these studies successfully improved the capacity of the physical model designed for seismic experiment simulation.

Further studies will focus on quality factor estimation to avoid the normalization calculation in the process and provide several sets of perfectly controlled experimental data to the scientific community. Moreover, the study of effective source reproducibility will be extended to the horizontal component that has become available recently in the MUSC laboratory (Valensi et al. 2015).

\section{ACKNOWLEDGEMENTS}

We would like to thank the CEA for the SEM3-D Spectral Element Method modelling code. We are also grateful to the CCIPL(Nantes, France) for providing access to its high-performance computing facilities and the support given by its staff. Finally, this study was carried out within the framework of the VIBRIS project (OSUNAIFSTTAR-Université de Nantes -CNRS) sponsored by the Paysde-la-Loire Region (France) and the French ANR project HIWAI ANR-16-CE31-0022-03 (October 2016 to September 2021). We would like to thank the reviewers for their valuable comments and suggestions to improve the quality of the paper.

\section{REFERENCES}

Aki, K. \& Richards, P.G., 2002. Quantitative Seismology, vol. 1, University Science Books.

Angona, F.A., 1960. Two-dimensional modeling and its application to seismic problems, Geophysics, 25(2), 468-482.

Auer, L., Nuber, A.M., Greenhalgh, S.A., Maurer, H. \& Marelli, S., 2013. A critical appraisal of asymptotic 3D-to-2D data transformation in fullwaveform seismic crosshole tomography, Geophysics, 78(6), R235-R247.

Ben-Hadj-Ali, H., Operto, S. \& Virieux, J., 2008. Velocity model building by 3D frequency-domain, full-waveform inversion of wide-aperture seismic data, Geophysics, 73(5), VE101-VE117.

Berkhout, A., Verschuur, D. \& Blacquiere, G., 2012. Illumination properties and imaging promises of blended, multiple-scattering seismic data: a tutorial, Geophys. Prospect., 60, 713-732.

Bishop, T., Bube, K., Cutler, R., Langan, R., Love, P., Resnick, J., Shuey, R. $\&$ Spinder, D., 1985. Tomographic determination of velocity and depth in laterally varying media, Geophysics, 50, 903-923.
Bodet, L., van Wijk, K., Bitri, A., Abraham, O., Cote, P., Grandjean, G. \& Leparoux, D., 2005. Surface-wave inversion limitations from laserDoppler physical modeling, J. Environ. Eng. Geophys., 10, 151-162.

Bohm, G., Carcione, J.M., Gei, D., Picotti, S. \& Michelini, A., 2015. Crosswell seismic and electromagnetic tomography for $\mathrm{CO} 2$ detection and monitoring in a saline aquifer, J. Pet. Sci. Eng., 133, 245-257.

Borisov, D. \& Singh, S.C., 2013. An efficient 3D elastic full waveform inversion of time-lapse seismic data using grid injection method, in 2013 SEG Annual Meeting, Society of Exploration Geophysicists.

Borisov, D. \& Singh, S.C., 2015. Three-dimensional elastic full waveform inversion in a marine environment using multicomponent ocean-bottom cables: a synthetic study, Geophys. J. Int., 201(3), 1215-1234.

Bretaudeau, F., Leparoux, D. \& Abraham, O., 2008. Small scale adaptation of the seismic full waveform inversion method - application to civil engineering applications, J. acoust. Soc. Am., 123(5).

Bretaudeau, F., Leparoux, D., Durand, O. \& Abraham, O., 2011. Small-scale modeling of onshore seismic experiment: A tool to validate numerical modeling and seismic imaging methods, Geophysics, 76(5), T101-T112.

Bretaudeau, F., Brossier, R., Leparoux, D., Abraham, O. \& Virieux, J., 2013. 2D elastic full-waveform imaging of the near-surface: application to synthetic and physical modelling data sets, Near Surf. Geophys., 11, 307-316.

Brossier, R., Operto, S. \& Virieux, J., 2009. Two-dimensional seismic imaging of the Valhall model from synthetic $\mathrm{OBC}$ data by frequency-domain elastic full-waveform inversion, in 2009 SEG Annual Meeting, Society of Exploration Geophysicists.

Brossier, R., Etienne, V., Hu, G., Operto, S. \& Virieux, J., 2013. Performances of 3D frequency-domain full-waveform inversion based on frequency-domain direct-solver and time-domain modeling: application to 3D OBC data from the Valhall field, presented at the International Petroleum Technology Conference, IPTC 16881.

Butzer, S., Kurzmann, A. \& Bohlen, T., 2013. 3D elastic full-waveform inversion of small-scale heterogeneities in transmission geometry, Geophys. Prospect., 61(6), 1238-1251.

Capdeville, Y., Guillot, L. \& Marigo, J.-J., 2010. 2-D non-periodic homogenization to upscale elastic media for $P-S V$ waves, Geophys. J. Int., 182(2), 903-922.

Castellanos, C., Etienne, V., Hu, G., Operto, S., Brossier, R. \& Virieux, J. et al., 2011. Algorithmic and methodological developments towards full waveform inversion in 3D elastic media, in 2011 SEG Annual Meeting, Society of Exploration Geophysicists.

Crase, E., Pica, A., Noble, M., McDonald, J. \& Tarantola, A., 1990. Robust elastic nonlinear waveform inversion: Application to real data, Geophysics, 55(5), 527-538.

Cristini, P. \& Komatitsch, D., 2012. Some illustrative examples of the use of a spectral-element method in ocean acoustics, J. acoust. Soc. Am., 131(3), EL229-EL235.

De Cacqueray, B., Roux, P., Campillo, M., Catheline, S. \& Boue, P., 2011. Elastic-wave identification and extraction through array processing: An experimental investigation at the laboratory scale, J. Appl. Geophys., 74, $81-88$.

Dhatt, G. \& Touzot, G., 1984. The Finite Element Method, Displayed, John Wiley \& Sons.

Etienne, V., Operto, S., Virieux, J. \& Jia, Y., 2010. Computational issues and strategies related to full waveform inversion in $3 \mathrm{D}$ elastic media: Methodological developments, in 2010 SEG Annual Meeting, Society of Exploration Geophysicists.

Favretto-Cristini, N., Tantsereva, A., Cristini, P., Ursin, B., Komatitsch, D. \& Aizenberg, A.M., 2014. Numerical modeling of zero-offset laboratory data in a strong topographic environment: results for a spectral-element method and a discretized Kirchhoff integral method, Earthq. Sci., 27(4), 391-399.

Festa, G. \& Vilotte, J., 2005. The Newmark as velocity-stress timestaggering: an efficient PML implementation for spectral element simulation of elastodynamics, Geophys. J. Int., 161, 798-812.

Forbriger, T., Gross, L. \& Schafer, M., 2014. Line-source simulation for shallow-seismic data. Part 1: theoretical background, Geophys. J. Int., 198(3), 1387-1404. 
French, W.S., 1974. Two-dimensional and three-dimensional migration of model-experiment reflection profiles, Geophysics, 39(3), 265-277.

Geuzaine, C. \& Remacle, J., 2009. GMSH: a three-dimensional finite element mesh generator with built-in pre- and post-processing facilities, Int. J. Numer. Methods Eng., 79(11), 1309-1331.

Groos, L., Schäfer, M., Forbriger, T. \& Bohlen, T., 2014. The role of attenuation in 2D full-waveform inversion of shallow-seismic body and Rayleigh waves, Geophysics, 79(6), R247-R261.

Guofeng, L., Yaning, L., Li, R. \& Xiaohong, M., 2013. 3D seismic reverse time migration on GPGPU, Comput. Geosci., 59, 10-23.

Hilterman, F., 1970. Three-dimensional seismic modeling, Geophysics, 35, $1020-1037$.

Howes, E., Tejada-Flores, L. \& Randolph, L., 1953. Seismic model study, J. acoust. Soc. Am., 25, 915-921.

Hulbert, G.M. \& Hughes, T.J., 1990. Space-time finite element methods for second-order hyperbolic equations, Comput. Methods Appl. Mech. Eng., 84(3), 327-348.

Köhn, D., Meier, T., Fehr, M., De Nil, D. \& Auras, M., 2016. Application of 2D elastic Rayleigh waveform inversion to ultrasonic laboratory and field data, Near Surf. Geophys., 14(5), 461-476.

Komatitsch, D. \& Vilotte, J.-P., 1998. The spectral element method: an efficient tool to simulate the seismic response of 2D and 3D geological structures, Bull. seism. Soc. Am., 88(2), 368-392.

Komatitsch, D., Vilotte, J.-P., Vai, R., Castillo-Covarrubias, J.M. \& SanchezSesma, F.J., 1999. The spectral element method for elastic wave equations-application to 2-D and 3-D seismic problems, Int. J. Numer. Methods Eng., 45(9), 1139-1164.

Komatitsch, D., Martin, R., Tromp, J., Taylor, M.A. \& Wingate, B.A., 2001. Wave propagation in $2 \mathrm{D}$ elastic media using a spectral element method with triangles and quadrangles, J. Comput. Acoust., 9(2), 703-718.

Komatitsch, D., Tsuboi, S. \& Tromp, J., 2005. The spectral-element method in seismology, in Seismic Earth: Array Analysis of Broadband Seismograms, pp. 205-227, eds Levander, A. \& Nolet, G., American Geophysical Union.

Lysmer, J. \& Drake, L.A., 1972. A finite element method for seismology, Methods Comput. Phys., 11, 181-216.

Martin, G.S., Wiley, R. \& Marfurt, K.J., 2006. Marmousi2: an elastic upgrade for Marmousi, Leading Edge, 25(2), 156-166.

Mo, Y., Greenhalgh, S.A., Robertsson, J.O. \& Karaman, H., 2015. The development and testing of a 2D laboratory seismic modelling system for heterogeneous structure investigations, J. Appl. Geophys., 116, 224-235.

Moczo, P., Kristek, J., Galis, M., Chaljub, E. \& Etienne, V., 2011. 3-D finitedifference, finite-element, discontinuous-Galerkin and spectral-element schemes analysed for their accuracy with respect to P-wave to $S$-wave speed ratio, Geophys. J. Int., 187(3), 1645-1667.

Morozov, I., 2004. Crustal scattering and some artefacts in receiver function images, Bull. seism. Soc. Am., 94, 1492-1499.

O’Brien, P. \& Symes, M., 1971. Model seismology, Rep. Prog. Phys., 34(2), 697-764.

Oliver, J., Press, F. \& Ewing, M., 1954. Two-dimensional model seismology, Geophysics, 19(2), 202-219.

Perez Solano, C., Donno, D. \& Chauris, H., 2014. Alternative waveform inversion for surface wave analysis in 2D media, Geophys. J. Int., 198, 1359-1372.

Plessix, R.-E., Baeten, G., de Maag, J.W., Klaassen, M., Rujie, Z. \& Zhifei, T., 2010. Application of acoustic full waveform inversion to a low-frequency large-offset land data set, in 2010 SEG Annual Meeting, Society of Exploration Geophysicists.

Pratt, R.G., 1999. Seismic waveform inversion in the frequency domain, Part 1: Theory and verification in a physical scale model, Geophysics, 64(3), 888-901.

Ravaut, C., Operto, S., Improta, L., Virieux, J., Herrero, A. \& Dell'Aversana, P., 2004. Multiscale imaging of complex structures from multifold wideaperture seismic data by frequency-domain full-waveform tomography: application to a thrust belt, Geophys. J. Int., 159(3), 1032-1056.

Rieber, F., 1936. Visual presentation of elastic wave patterns under various structural conditions, Geophysics, 1, 196-218.
Romdhane, A., Grandjean, G., Brossier, R., Rejiba, F., Operto, S. \& Virieux, J., 2011. Shallow-structure characterization by $2 \mathrm{D}$ elastic full-waveform inversion, Geophysics, 76(3), R81-R93.

Schafer, M., Gross, L., Forbriger, T. \& Bohlen, T., 2014. Line-source simulation for shallow-seismic data. Part 2: Full-waveform inversion-a synthetic 2-D case study, Geophys. J. Int., 198, 1405-1418.

Seron, F.J., Sanz, F.J., Kindelan, M. \& Badal, J.I., 1990. Finite-element method for elastic wave propagation, Commun. Appl. Numer. Methods, 6, 359-368.

Shipp, R.M. \& Singh, S.C., 2002. Two-dimensional full wavefield inversion of wide-aperture marine seismic streamer data, Geophys. J. Int., 151(2), 325-344.

Tromp, J., Komattisch, D. \& Liu, Q., 2008. Spectral-element and adjoint methods in seismology, Commun. Comput. Phys., 3(1), 1-32.

Valensi, R., Leparoux, D., Durand, O., Bretaudeau, F. \& Côte, P., 2015. Multicomponent reduced scale seismic modelling: upgrade of the MUSC laboratory with application to polarization observations, Geophys. J. Int., 202(3), 1993-2024.

Van Wijk, K., 2005. On estimating the impulse response between receivers in a controlled ultrasonic experiment, Geophysics, 71, SI79-SI84.

Virieux, J. \& Operto, S., 2009. An overview of full-waveform inversion in exploration geophysics, Geophysics, 74(6), WCC1-WCC26.

Wang, Y. \& Rao, Y., 2009. Reflection seismic waveform tomography, J. Geophys. Res., 114, B03304, doi:10.1029/2008JB005916.

Wong, J., Hall, K.W., Gallant, E.V., Maier, R., Bertram, M. \& Lawton, D.C., 2009. Seismic physical modeling at university of calgary, CSEG Recorder, 34(3), 36-43.

\section{APPENDIX: 3-D/2-D DIFFERENCES}

In this appendix we shall describe 3-D-2-D spreading corrections proposed by Forbriger et al. (2014): single velocity, direct wave and hybrid methods.

The displacement field $u$ can be evaluated at position $(\boldsymbol{x}=$ $(x, y, z))$ and time $(t)$ by (Aki \& Richards 2002):

$u(\boldsymbol{x}, t)=\int_{-\infty}^{+\infty} \mathrm{d} \tau \iiint_{V} G(\boldsymbol{\xi}, t-\tau ; \boldsymbol{x}, 0) f(\boldsymbol{\xi}, \tau) \mathrm{d} V(\boldsymbol{\xi})$,

where $G(\boldsymbol{\xi}, t-\tau ; \boldsymbol{x}, 0)$ is the Green's function between the source location $(\boldsymbol{\xi})$ and the observation point $(\boldsymbol{x})$, and $f(\boldsymbol{\xi}, \tau)$ is the seismic source function. The body force distribution for a point source $\left(f_{P}\right)$ and a line source $\left(f_{L}\right)$, located at positions $\boldsymbol{x}_{s}=\left(x_{s}, y_{s}, z_{s}\right)$ and $\boldsymbol{x}_{\boldsymbol{s} 2 \boldsymbol{D}}=\left(x_{s}, z_{s}\right)$, respectively, in a 2-D structure invariant along the $y$-axis are as follows:

$f_{P}\left(\boldsymbol{x}, t ; \boldsymbol{x}_{\boldsymbol{s}}\right)=\boldsymbol{F}(t) \delta_{\boldsymbol{x}}\left(x-x_{s}\right) \delta_{\boldsymbol{x}}\left(y-y_{s}\right) \delta_{\boldsymbol{x}}\left(z-z_{s}\right)$,

$f_{L}\left(x, t ; \boldsymbol{x}_{\boldsymbol{s} 2 \boldsymbol{D}}\right)=\boldsymbol{F}(t) C \delta_{\boldsymbol{x}}\left(x-x_{s}\right) \delta_{\boldsymbol{x}}\left(z-z_{s}\right)$,

where $C$ is a constant, $(\boldsymbol{F}(t))$ is the wavelet time function and $(\delta)$ is the Dirac function. Substituting eq. (A2) into eq. (A1) and eq. (A3) into eq. (A1) leads to the wave motion due to a point source $u_{P}$ and the wave motion due to a line source $u_{L}$

$u_{P}\left(\boldsymbol{x}, t ; \boldsymbol{x}_{s}\right)=\int_{-\infty}^{+\infty} G\left(\boldsymbol{x}_{\boldsymbol{s}}, t-\tau ; \boldsymbol{x}\right) F(\tau) \mathrm{d} \tau$

$u_{L}\left(\boldsymbol{x}, t ; \boldsymbol{x}_{\boldsymbol{s}}^{2 D}\right)=\int_{-\infty}^{+\infty} \int_{-\infty}^{+\infty} G\left(\boldsymbol{x}_{s}^{2 D}, t-\tau ; \boldsymbol{x}\right) F(\tau) C \mathrm{~d} y^{\prime} \mathrm{d} \tau$.

The equivalent displacement $u_{L}$ for a line source can be obtained by the displacement field $u_{P}$ generated by a point source by integration 
along $y$ :

$u_{L}\left(x, y, z, t ; x_{s}, z_{s}\right)=\int_{-\infty}^{+\infty} u_{P}\left(x, y, z, t-\tau ; x_{s}, y^{\prime}, z_{s}, 0\right) C \mathrm{~d} y^{\prime}$.

Eq. (A6) shows that in terms of amplitude, the displacement generated by a line source is greater than the displacement generated by a point source. Taking $g_{k}^{3 D}(r)$ and $g_{k}^{2 D}(r)$, the Fourier transform of the 3-D and 2-D Green's function in the acoustic approximation, respectively, with $k$ being the wavenumber and $r=\left|\boldsymbol{x}-\boldsymbol{x}_{\boldsymbol{s}}\right|$ the source-receiver offset. In the far-field approximation, Forbriger et al. (2014) demonstrate:

$\lim _{r \rightarrow \infty} \frac{g_{k}^{2 D}(r)}{g_{k}^{3 D}(r)} \approx \sqrt{\frac{2 \pi r}{k}} \cdot e^{i \frac{\pi}{4}}$

Replacing the wavenumber $k=\omega / v_{p h}$, where $\omega$ is the angular frequency and $v_{\mathrm{ph}}$ is the phase velocity, results in

$\sqrt{2 \pi r v_{\mathrm{ph}}} \cdot \sqrt{\frac{\pi}{\omega}} e^{i \frac{\pi}{4}}=F_{\mathrm{amp}} \cdot \widetilde{F}_{\sqrt{t^{-1}}}$, where $F_{\text {amp }}=\sqrt{2 \pi r v_{\mathrm{ph}}}$ is the amplitude factor and $\widetilde{F}_{\sqrt{t^{-1}}}$ applies the phase shift. It results in

$u_{L}(r, \omega)=u_{P}(r, \omega) \cdot F_{\mathrm{amp}} \cdot \widetilde{F}_{\sqrt{t^{-1}}}$.

This correction is called the single-velocity transformation which is recommended, after numerical tests (Forbriger et al. 2014; Schafer et al. 2014), for small offsets. For a larger offset, by stating that the offset is almost equal to the propagation distance, Schafer et al. (2014) propose to replace the previous amplitude factor with

$F_{\text {amp }}=r \sqrt{\frac{2 \pi}{t}}$.

The resulting correction is called the direct-wave transformation. Finally, the hybrid method proposed by Forbriger et al. (2014) and Schafer et al. (2014) consists in using the two previous transformations with a smoothly offset conditioned transition from the nearfield to the far-field.

The hybrid method was successfully validated by Schafer et al. (2014) in numerical modelling and reconstruction tests with a 2-D FWI method and 3-D numerical data generated in a 2-D structure. 